\title{
1q21.1 Microduplication expression in adults
}

\author{
Alessia Dolcetti, BSc${ }^{1}$, Candice K. Silversides, MD²,3, Christian R. Marshall, PhD ${ }^{4,5}$, Anath C. Lionel, \\ $\mathrm{MSc}^{4,5}$, Dimitri J. Stavropoulos, PhD ${ }^{6,7}$, Stephen W. Scherer, $\mathrm{PhD}^{4,5}$ and Anne S. Bassett, MD ${ }^{1,2,8}$
}

\begin{abstract}
Purpose: Rare, recurrent chromosome 1q21.1 duplications have been associated with developmental delay, congenital anomalies, and macrocephaly in children. Data on adult clinical expression would help to inform genetic counseling.
\end{abstract}

Methods: A systematic review of 22 studies reporting 107 individuals (59 children and 48 adults) with 1q21.1 duplications was conducted. We compiled the available phenotypic data to attempt to identify the most highly associated clinical features and to determine expression in adults. We also report on seven adult cases newly identified in the studies of schizophrenia and tetralogy of Fallot at our center.

Results: Five cases were ascertained as controls, 32 as relatives of probands, and 70 as having clinical features: autism spectrum disorder $(n=15)$, congenital heart disease $(n=12)$, schizophrenia $(n=10)$, or other, mostly developmental, features $(n=33)$. The 1q21.1 duplication was significantly enriched in the cohorts with schizophrenia $(P=0.0155)$ and tetralogy of Fallot $(P=0.0040)$ at our center as compared with controls. There was a paucity of clinical data for adults; the most common features, other than those used for ascertainment, included macrocephaly and abnormalities of possible connective tissue origin (e.g., carpal tunnel syndrome).

Conclusion: Further data are needed to characterize lifetime expression of 1q21.1 duplications. These initial results, however, suggest that anticipatory care should include attention to later-onset conditions such as schizophrenia.

Genet Med 2013:15(4):282-289

Key Words: 1q21.1 duplication; copy-number variation; macrocephaly; schizophrenia; tetralogy of Fallot

\section{INTRODUCTION}

The chromosome 1q21.1 locus is a complex region with multiple low-copy repeats that make the region susceptible to recurrent deletions and duplications. Large rare copy-number variants (CNVs) at this locus have recently been associated with genomic disorders (OMIM nos. 612474 and 612475). Two main classes of 1q21.1 CNVs, spanning 20-40 genes, have been described. ${ }^{1}$ The more common one (class I, $\sim 1.8 \mathrm{Mb}$ ) includes only the distal 1q21.1 region, whereas the longer one (class II, $\sim 2.7 \mathrm{Mb}$ ) extends proximally to encompass the thrombocytopenia absent radius syndrome region (Figure 1). Brunetti-Pierri et al. ${ }^{1}$ reported both CNV classes among their patients, with no obvious relationship between duplication/ deletion size and severity of symptoms. Although incomplete penetrance and variable expressivity are common to deletions and duplications at the 1q21.1 locus, initial reports have suggested that the deletion has a relatively more consistent pattern of expression including microcephaly, developmental abnormalities, and schizophrenia. ${ }^{1,2}$ The 1q21.1 duplication was the 11th most common of 28 genomic disorders reported in a recent laboratory survey of clinical microarray results. ${ }^{3}$ Consistent with the general tendency for duplications to be less deleterious than deletions and a corresponding ascertainment bias in clinical samples, ${ }^{4}$ the 1q21.1 duplication may be rarer than the reciprocal deletion in many, but not all, clinical settings.

Although the 1q21.1 duplication has been associated with macrocephaly, developmental delay, autism spectrum disorder, and congenital anomalies in children, little is known about its expression in adults. ${ }^{1,2}$ Other genomic disorders (e.g., 22q11.2 deletion syndrome) have an adult phenotype that differs from the pediatric phenotype. Growing use of clinical genome-wide microarrays to investigate CNVs has meant increasing pressure for more information on the lifetime expression of 1q21.1 duplications to better inform individuals found to have this $\mathrm{CNV}$, their families, and clinicians. In this study, we comprehensively reviewed all reports of 1q21.1 duplications to date, focusing on lifetime expression. We hypothesized that, due to the ascertainment of many adults with 1q21.1 duplications as transmitting parents, they would have similar or milder physical phenotypes and milder neuropsychiatric phenotypes as compared with children.

\section{Literature review}

MATERIALS AND METHODS

We attempted to identify reports of all 1q21.1 duplication cases published by 31 December 2011. We first used PubMed and the search terms "1q21 AND (duplication OR gain OR copy

\footnotetext{
${ }^{1}$ Clinical Genetics Research Program, Centre for Addiction and Mental Health, Toronto, Ontario, Canada; ${ }^{2}$ Division of Cardiology, Department of Medicine, University Health Network, Toronto, Ontario, Canada; ${ }^{3}$ Toronto Congenital Cardiac Centre for Adults, Toronto General Hospital, Toronto, Ontario, Canada; ${ }^{4}$ The Centre for Applied Genomics and Program in Genetics and Genome Biology, The Hospital for Sick Children, Toronto, Ontario, Canada; ${ }^{5}$ Department of Molecular Genetics and McLaughlin Centre, University of Toronto, Toronto, Ontario, Canada; ${ }^{6}$ Cytogenetics Laboratory, Department of Pediatric Laboratory Medicine, Hospital for Sick Children, Toronto, Ontario, Canada; ${ }^{7}$ Department of Laboratory Medicine and Pathology, University of Toronto, Toronto, Ontario, Canada; ${ }^{8}$ Department of Psychiatry, University of Toronto, Toronto, Ontario, Canada. Correspondence: Anne S. Bassett (anne.bassett@utoronto.ca)
} 
number variant)".1,2,5-12 We also examined primary articles and reviews of CNVs in autism, ${ }^{13}$ schizophrenia ${ }^{14}$ mental retardation, ${ }^{15}$ and congenital heart disease ${ }^{16}$ for references to studies reporting subjects with 1q21.1 duplications. ${ }^{17-20}$ Other citations involving 1q21.1 duplication cases were also assessed. ${ }^{21-34}$ Cases of 1q21.1 duplication in GeneReviews (http://www.ncbi.nlm. nih.gov/books/NBK52787) and the four references provided therein ${ }^{1,2,10,25}$ were reviewed as well, but no new subjects or phenotypic data were identified. We could not include individuals from two large clinical laboratory surveys because the number of adults was not specified and many subjects may have overlapped the cases already identified. ${ }^{3,35}$

We systematically reviewed 28 primary reports identified and their accompanying supplemental materials to identify unique individuals and abstract as much information as possible on each subject. ${ }^{1,2,5-12,17-34}$ Any subjects with 1q21.1 duplications that were not $1.0-5.0 \mathrm{Mb}$ (Figure 1) in size were excluded, ${ }^{12,31}$ allowing us to include atypical variants that were larger or smaller than the standard duplication classes used in our analyses. We documented the ascertainment criteria, country of origin, authors, and the demographic, clinical, and genotypic characteristics of subjects in different studies, and used these variables to attempt to identify duplicate cases reported in two or more papers. Four studies were excluded because the sources of subjects overlapped with those of other reports and/or the origin of cases was not specified. . $^{17,18,32,34}$ We compiled data for 107 subjects from the remaining 22 studies ${ }^{1,2,5-11,19-30,33}$ for the main analyses in this report.

Individuals were classified as adults ( $\geq 18$ years) or children $(<18$ years). Because a specific age was not provided for 70 of the 107 cases (65.4\%), several assumptions had to be made to categorize these subjects as children or adults by inference. Probands and siblings reported in pediatric journals were assumed to be children. All transmitting parents were assumed to be adults, as were subjects who were ascertained with schizophrenia, bipolar disorder, or depression. Sex was not specified for 58 of 107 subjects (54.2\%); however, we were able to use sex-specific medical conditions (e.g., hypospadias) or published photographs to infer sex in seven of these cases. To be as inclusive as possible, clinical information was defined as the description of any physical, cognitive, developmental, or neurological features other than sex, age, or the feature used for ascertainment. The report of individuals as "normal" or "affected" was only considered clinical information when it was clear which features were being described. For cases from our center and the literature, macrocephaly and tall stature were defined as physical measurements $\geq 90$ th percentile, and these were assessed using sex-matched head circumference charts

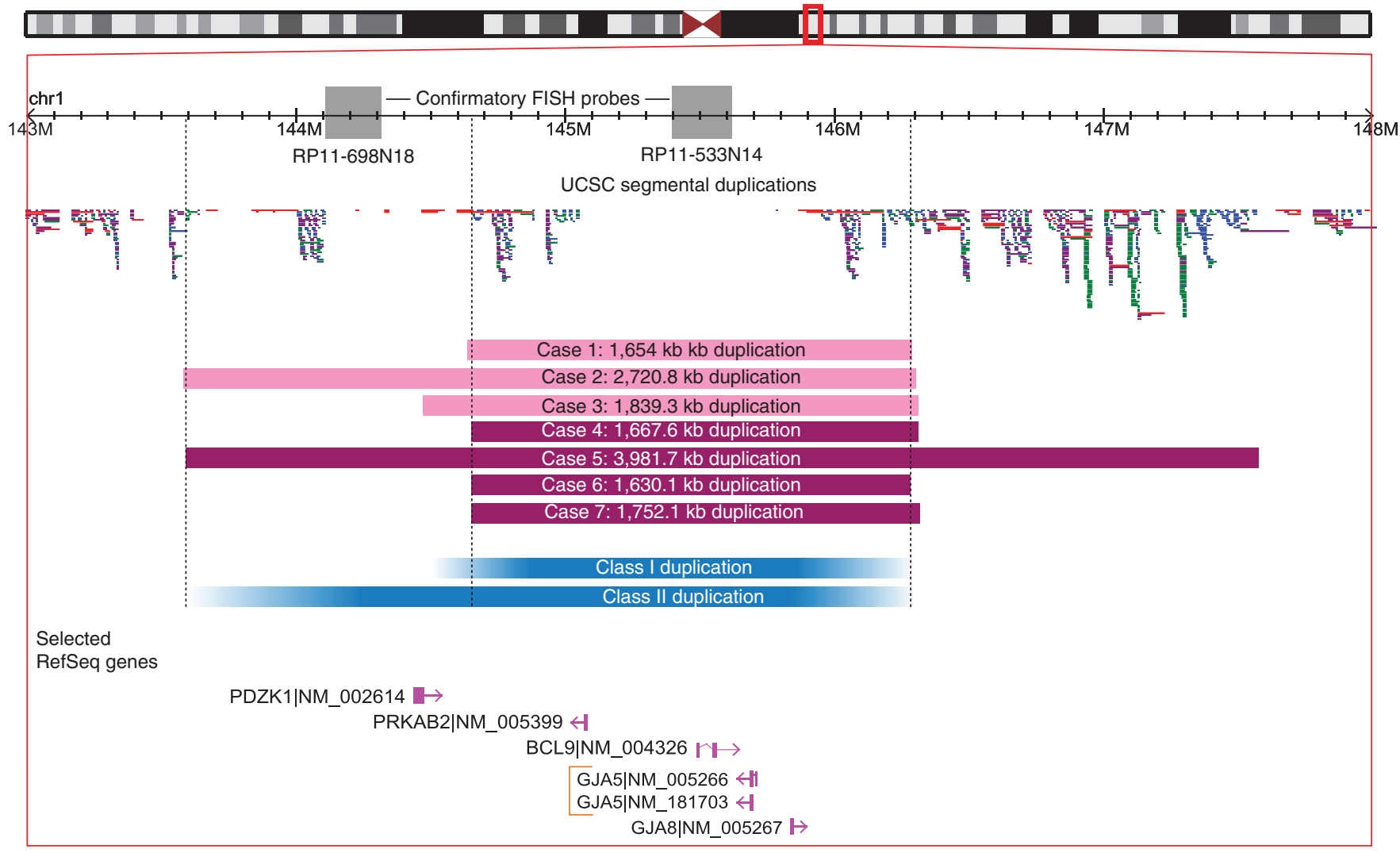

UCSC Genome Browser, Human Mar. 2006 Assembly

Figure 1 Large, rare duplications in the 1q21.1 region detected in cohorts with schizophrenia (pink bars) and tetralogy of Fallot (purple bars). Blue bars and dashed vertical lines represent duplication classes I and II. Genomic parameters are from NCBI Build 36. Selected genes that are shown are potentially implicated in congenital heart disease and schizophrenia. 
that correct for height ${ }^{36}$ and height-for-age data tables from the Centers for Disease Control and Prevention (http://www.cdc. gov/growthcharts). Body mass index was calculated using the formula weight $(\mathrm{kg}) /$ height $(\mathrm{m})^{2}$, where body mass index $\geq 30.0$ was defined as obesity. Cases with macrocephaly, tall stature, and/or obesity were considered to have a possible overgrowthlike phenotype.

Duplication class I or $\mathrm{II}^{1}$ was assigned to cases in which microarray coordinates were provided. All transmitting parents were assumed to have the same duplication as their affected child. Information on other genome-wide rare CNVs was not available for the vast majority of cases.

\section{Adults ascertained through studies at our center}

The sample population for identification of 1q21.1 duplications at our center comprised 502 unrelated adults with tetralogy of Fallot (TOF) seen at the Toronto Congenital Cardiac Centre for Adults ${ }^{37}$ and 463 unrelated adults with a Diagnostic and Statistical Manual of Mental Disorders 4th Edition (DSM-IV) diagnosis of chronic schizophrenia or schizoaffective disorder ascertained mainly through four eastern Canadian clinics. ${ }^{40}$ Lifetime medical history and physical features were directly assessed by researchers blind to genotype using a standard protocol. ${ }^{38}$ Medical records and available psychiatric records were reviewed for all participants. Subjects were genotyped on the Affymetrix Genome-Wide Human SNP Array 6.0 (Santa Clara, CA), and CNV analysis was performed using standard methods described elsewhere..$^{39}$ The control population consisted of 2,357 individuals of European ancestry from the PopGen project $(n=1,123)$ and Ottawa Heart Institute $(n=1,234)$ genotyped on the Affymetrix SNP array 6.0. ${ }^{39}$ Cases of 1q21.1 duplication were confirmed using quantitative PCR and/or clinical microarrays. ${ }^{37,40}$

\section{Analyses}

Clinical data on adults from our center and the literature were compiled to identify features present in both populations. Any feature present in two or more subjects from our center or the literature was considered to be possibly associated with the 1q21.1 duplication phenotype. Statistical analyses were performed using SAS software (version 9.1; SAS Institute, Cary, $\mathrm{NC}$ ). Comparisons using categorical data were made using a $\chi^{2}$ or Fisher's exact test where appropriate. All analyses were twotailed, with statistical significance defined as $P<0.05$. Odds ratios and $95 \%$ confidence intervals were used to assess the association between 1q21.1 duplications and schizophrenia or TOF, as compared with controls.

\section{RESULTS}

There were 107 individuals (59 children and 48 adults) with 1q21.1 duplications from 76 families identified from the literature reviewed (Supplementary Table S1 online). Of the 31 relatives with 1q21.1 duplications, 26 were transmitting parents and five were siblings. The 76 probands (including adults and children) comprised 70 subjects ascertained because of major clinical features, five reported in nonoverlapping control populations, ${ }^{2,5,6}$ and one parent of a child with autism spectrum disorder where the child did not have a 1q21.1 duplication. ${ }^{22}$ The majority were referred from centers in Europe or the United States. Of the 70 clinically ascertained probands, 15 (21.4\%) were ascertained with autism spectrum disorder, 12 (17.1\%) with congenital heart disease, 10 (14.3\%) with schizophrenia, two $(2.9 \%)$ with bipolar disorder, and one $(1.4 \%)$ with recurrent depressive disorder. The remaining 30 (42.9\%) probands were recruited with one or more developmental features (intellectual disability, developmental delay, autism spectrum disorder, congenital anomalies, and/or dysmorphic features) where the precise features were not specified for individuals. Cases of 1q21.1 duplication were reported as inherited in 31 cases, de novo in 16, and of unknown origin in 60 . Of the $60(78.9 \%)$ probands for whom duplication class was reported, 56 (93.3\%; 36 children and 20 adults) were class I and 4 (6.7\%; 2 children and 2 adults) were class II.

Duplications at 1q21.1 are rare in control populations. Soemedi et al. ${ }^{5}$ identified 1q21.1 duplications in 3 of 10,910 $(0.027 \%)$ individuals originating from multiple source cohorts. Two were from the Wellcome Trust Case-Control Consortium 2 control cohort of 5,919 $(0.034 \%)$ adults from the 1958 British Birth Cohort and the UK Blood Service (http://www.wtccc.org. $\mathrm{uk} / \mathrm{ccc} 2 /)$, and the third individual with a 1q21.1 duplication was from a cohort of 2,026 (0.049\%) children seen at the Children's Hospital of Philadelphia. ${ }^{24}$ There were no 1q21.1 duplications in the remaining 2,965 controls from other sources. ${ }^{5} \mathrm{~A}$ duplication at the 1q21.1 locus was also reported by Mefford et al. ${ }^{2}$ in 1 of $4,737(0.021 \%)$ controls, where the variant was detected in a subgroup of 1,190 individuals randomly recruited from lists of residential telephone numbers and tax assessment rolls in Canada. ${ }^{21}$ The International Schizophrenia Consortium detected a 1q21.1 duplication in 1 of 3,181 (0.031\%) controls from various European sites and sources; ${ }^{6}$ the origin of individual controls was not specified. In two of the four control populations with reported 1q21.1 duplications, participants were not screened for disease before enrollment.

\section{Children with 1q21.1 duplications}

There were 59 children (19 (32.2\%) male, 8 (13.6\%) female, and 32 (54.2\%) sex not reported) with 1q21.1 duplications identified from the literature reviewed. Although a greater proportion of children were reported to be male than female, the sex distribution was not significantly different from the expected 1:1 ratio $\left(\chi^{2}=2.34, \mathrm{df}=1, P=0.1263\right)$. Of these 59 children (median age 5, range $0.3-16$ years for 31 with age provided), clinical data beyond ascertainment features were available for $43(72.9 \%)$ cases. Including ascertainment criteria and multiple features per subject, $30(50.8 \%)$ had an intellectual disability or developmental delay, 21 (35.6\%) had autism or autistic features, $20(33.9 \%)$ had dysmorphic facial features, 12 (20.3\%) had congenital heart disease (eight TOF, two ventricular septal defect, one univentricular heart, and one "complex"), five (8.5\%) had seizures, four (6.8\%) had a failure to thrive, and two (3.4\%) 
had attention deficit hyperactivity disorder. Of 26 (44.1\%) children with head circumference results available, $16(61.5 \%)$ had macrocephaly. Weight was above the 90th percentile in two of six cases with data. Of seven cases describing height, one had tall stature. Other features recorded in two or more cases were hypotonia $(n=5)$, hypertonia $(n=3)$, frontal bossing $(n=3)$, high palate $(n=2)$, esotropia $(n=2)$, low-set ears $(n=3)$, downslanting palpebral fissures $(n=2)$, hypertelorism $(n=2)$, small chin $(n=2)$, gastroesophageal reflux $(n=2)$, and hypospadias $(n=2)$.

\section{Adults with 1q21.1 duplications}

There were 48 adults (13 (27.1\%) male, 16 (33.3\%) female, and 19 (39.6\%) sex not reported) with 1q21.1 duplications identified from the literature, with median age 29 and range 19-57 years for the six (12.5\%) with age reported. Of these 48 adults, 27 (56.3\%; 12 male, 13 female, and 2 unknown sex) were ascertained as parents of children with clinical features, $10(20.8 \%)$ with schizophrenia, 4 (8.3\%; 1 male and 3 female) with TOF, 2 (4.2\%) with bipolar disorder, 1 (2.1\%) with recurrent depressive disorder, and $4(8.3 \%)$ as controls. Clinical data beyond ascertainment features were available for $20(41.7 \%)$ adults from eight reports (Table 1, cases $8-27), 1,5,7,10,11,22,23,29$ a significantly smaller proportion than for children $\left(\chi^{2}=10.65, \mathrm{df}=1, P=\right.$ 0.0011 ). Of these 20 adults ( 8 male and 12 female), 16 (80.0\%) were ascertained as parents of children with clinical features and $4(20.0 \%)$ with TOF. Even when clinical information was available, few details were provided on the adult phenotype. In 12 cases, this was limited to features described as normal (e.g., "normal cognition") ${ }^{10}$ or absent (e.g., "no extracardiac phenotype"). ${ }^{5}$

At our center, we detected 1q21.1 duplications in seven ( 2 male and 5 female) unrelated Caucasian adult probands (median age 51, range 28-62 years) (Table 1, cases 1-7; Figure 1), three ascertained with schizophrenia ${ }^{40}$ and four with TOF. $^{37}$ There were no 1q21.1 duplications in 2,357 adult controls, thus the CNV was enriched in both the schizophrenia (odds ratio 15.4 (95\% confidence interval 1.6-148.0); $P=$ $0.0155)$ and TOF (odds ratio 18.9 (95\% confidence interval 2.1-169.7); $P=0.0040$ ) cohorts studied.

Tables 1 and 2 show the demographic and clinical data available for 27 adults (10 males and 17 females), seven (25.9\%) from our center and $20(74.1 \%)$ from the literature reviewed. Three (21.4\%) of 14 adults with data were reported to have some dysmorphic facial features, two described as mild. Of the 13 adults with any data on height, weight, or head circumference, nine met criteria for a possible overgrowth phenotype. Eight (61.5\%) of the 13 adults for whom head circumference results were available had macrocephaly. Three of seven adults with data met criteria for obesity and three of seven had tall stature. Other conditions present in two or more subjects were cysts $(n=4)$, hypercholesterolemia $(n=3)$, type 2 diabetes mellitus $(n=3)$, cubitus valgus $(n=3)$, carpal tunnel syndrome $(n=2)$, deep vein thrombosis $(n=2)$, cholelithiasis $(n=2)$, and cataracts $(n=2)$. Of those with any neuropsychiatric data available ( 15 of 20 adults reviewed and 7 from our center), two subjects had anxiety and/or depression, one had bipolar disorder, and one had childhood attention deficit hyperactivity disorder, in addition to the three ascertained with schizophrenia. Four of 16 adults with data on cognitive functioning had learning disabilities; none of the 22 with neuropsychiatric data were reported to have been diagnosed with intellectual disability, autism spectrum disorder, or seizures. There was no evidence for the adults ascertained at our center that the severity of the neuropsychiatric and developmental phenotypes that would have been observable when they were children had improved with age.

Including the seven from our center, the adults with sex known comprised 15 males and 21 females. Females outnumbered males among the adult probands ascertained with disease ( 3 male and 8 female) and parents of children with clinical features (12 male and 13 female). There was a significantly greater proportion of reported females than males among adults as compared with children $\left(\chi^{2}=5.12, \mathrm{df}=1, P=0.0237\right)$; however, the sex ratio in the adults was not statistically different from an expected $1: 1$ ratio $\left(\chi^{2}=0.50, \mathrm{df}=1, P=0.4782\right)$.

\section{DISCUSSION}

We reviewed the available phenotypic and genotypic data for 107 subjects with 1q21.1 duplications, including 48 adults. This review is the largest of its kind to date. Review of the existing literature for 107 reported cases of 1q21.1 duplications revealed limited clinical information on this genomic disorder in 48 adults, with some phenotypic data (other than ascertainment features) available for only 20 individuals, most of whom were transmitting parents. Where sex was reported, a significantly greater proportion of adults as compared with children were female. The sex ratios in children and adults, although skewed in opposite directions, were not significantly different from the expected 1:1 ratio. Ascertainment may have played a role; a greater proportion of adults as compared with children were female, possibly because most children with sex reported were ascertained with conditions more common in males (e.g., $\sim 30 \%$ with autism spectrum disorder), whereas most adults were transmitting parents. We also found that inheritance status of the 1q21.1 duplication was determined for a minority of individuals and, where known, was almost twice as likely to be inherited as of de novo origin, similar to laboratory survey results for duplications and deletions at this locus. ${ }^{35}$

Collective data on the 20 adults from our literature review and the seven from our center suggested that although 1q21.1 duplication expression is variable, there may be an emerging pattern of associated features. Since the first report of a 1q21.1 duplication in a TOF proband, ${ }^{23}$ several studies have now reported that $1 \mathrm{q} 21.1$ duplications are enriched in TOF at remarkably similar prevalences: $0.78 \%,{ }^{10} 0.84 \%,{ }^{5}$ and $0.80 \% .{ }^{37}$ Prevalence results for schizophrenia, autism spectrum disorder, and intellectual disability, although elevated, are less clear. ${ }^{2,9}$ Although numbers were small, the majority of children (16 of 26) and adults ( 8 of 13) with head circumference 
Table 1 Major clinical features of 27 adults with 1q21.1 duplications

\begin{tabular}{|c|c|c|c|c|c|c|c|c|c|c|}
\hline Case & Ascertainment & Sex & Age & Class $^{\mathrm{a}}$ & $\begin{array}{l}\text { OFC } \\
\text { (percentile) }\end{array}$ & $\begin{array}{l}\text { Height } \\
\text { percentile }\end{array}$ & BMI & Dysmorphic & Neuropsychiatric ${ }^{b}$ & Cardiovascularb \\
\hline \multicolumn{11}{|c|}{ Cases identified at our center $(n=7)$} \\
\hline 1 & SZ & $\mathrm{F}$ & 46 & I & $\begin{array}{l}\text { Normal } \\
(<50)\end{array}$ & $50-75$ & 28.2 & No & (SZ), migraines & Deep vein thrombosis \\
\hline $2^{c}$ & SZ & $\mathrm{F}$ & 62 & $\|$ & $\begin{array}{l}\text { Macrocephaly } \\
(>90)\end{array}$ & $90-95$ & 30.9 & Mild ${ }^{d}$ & $\begin{array}{l}\text { (SZ), LD, malignant } \\
\text { neuroleptic syndrome }\end{array}$ & $\begin{array}{l}\text { Deep vein } \\
\text { thrombosis, varicose } \\
\text { veins }\end{array}$ \\
\hline 4 & TOF & $\mathrm{F}$ & 28 & I & $\begin{array}{l}\text { Normal } \\
(>50)\end{array}$ & 97 & 19.6 & No & Severe migraines & (TOF) \\
\hline 5 & TOF & $\mathrm{F}$ & 34 & ॥ & $\begin{array}{l}\text { Macrocephaly } \\
(>90)\end{array}$ & $90-95$ & 37.9 & No & $\begin{array}{l}\text { Depression, anxiety, } \\
\text { vertigo }\end{array}$ & (TOF) \\
\hline 6 & TOF & $\mathrm{F}$ & 59 & 1 & $\begin{array}{l}\text { Macrocephaly } \\
(>90)\end{array}$ & 75 & 31.4 & No & None & $\begin{array}{l}\text { (TOF), hypertension, } \\
\text { venous stasis ulcers }\end{array}$ \\
\hline
\end{tabular}

Literature $\operatorname{cases}^{\dagger}(n=20)$

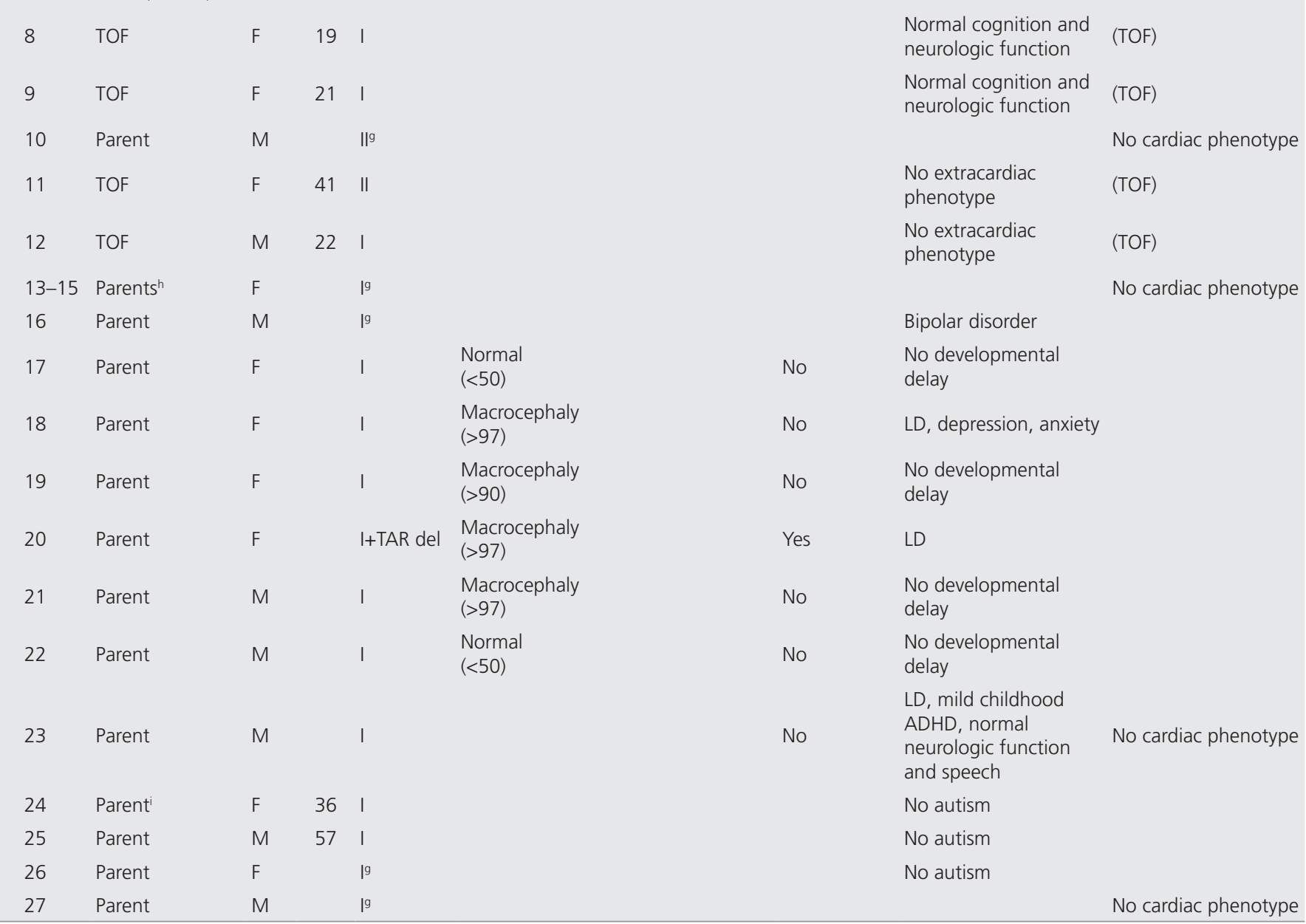

All 48 adults reported in 22 sources from the literature are included in Supplementary Table $\mathbf{S 1}$ online.

ADHD, attention deficit hyperactivity disorder; BMI, body mass index; del, deletion; empty cells, unknown; F, female; LD, learning disability; M, male; OFC, orbitofrontal circumference; SZ, schizophrenia; TAR, thrombocytopenia with absent radius region; TOF, tetralogy of Fallot.

${ }^{a}$ Cases 2, 5, and 9 had 1q21.1 duplications of de novo origin. bParentheses indicate features used for case ascertainment. cFamily history of schizophrenia. ${ }^{\mathrm{d}} \mathrm{Case} 2 \mathrm{had}$ down-slanted palpebral features, a slightly bulbous tipped nose, mild frontal bossing, and an overfolded helix of one ear. ${ }^{\text {e}}$ Case 7 had narrow-slanted palpebral features, a bulbous tipped nose, low-set ears, and a high-arched narrow palate. 'Cases 8-10 were reported by Greenway et al., 2009 (ref. 10); 11-15 by Soemedi et al., 2012 (ref. 5); 16 by Grozeva et al., 2010 (ref. 29); 17-22 by Brunetti-Pierri et al., 2008 (ref. 1); 23 by Harvard et al., 2011 (ref. 7); $24-25$ by Sanders et al., 2011 (ref. 22 ); 26 by Pinto et al.,

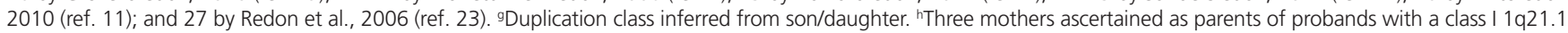
duplication. The mother of a child with autism spectrum disorder where the child did not have a 1q21.1 duplication. 
Table 2 Other medical conditions reported in 11 adults with 1q21.1 duplications

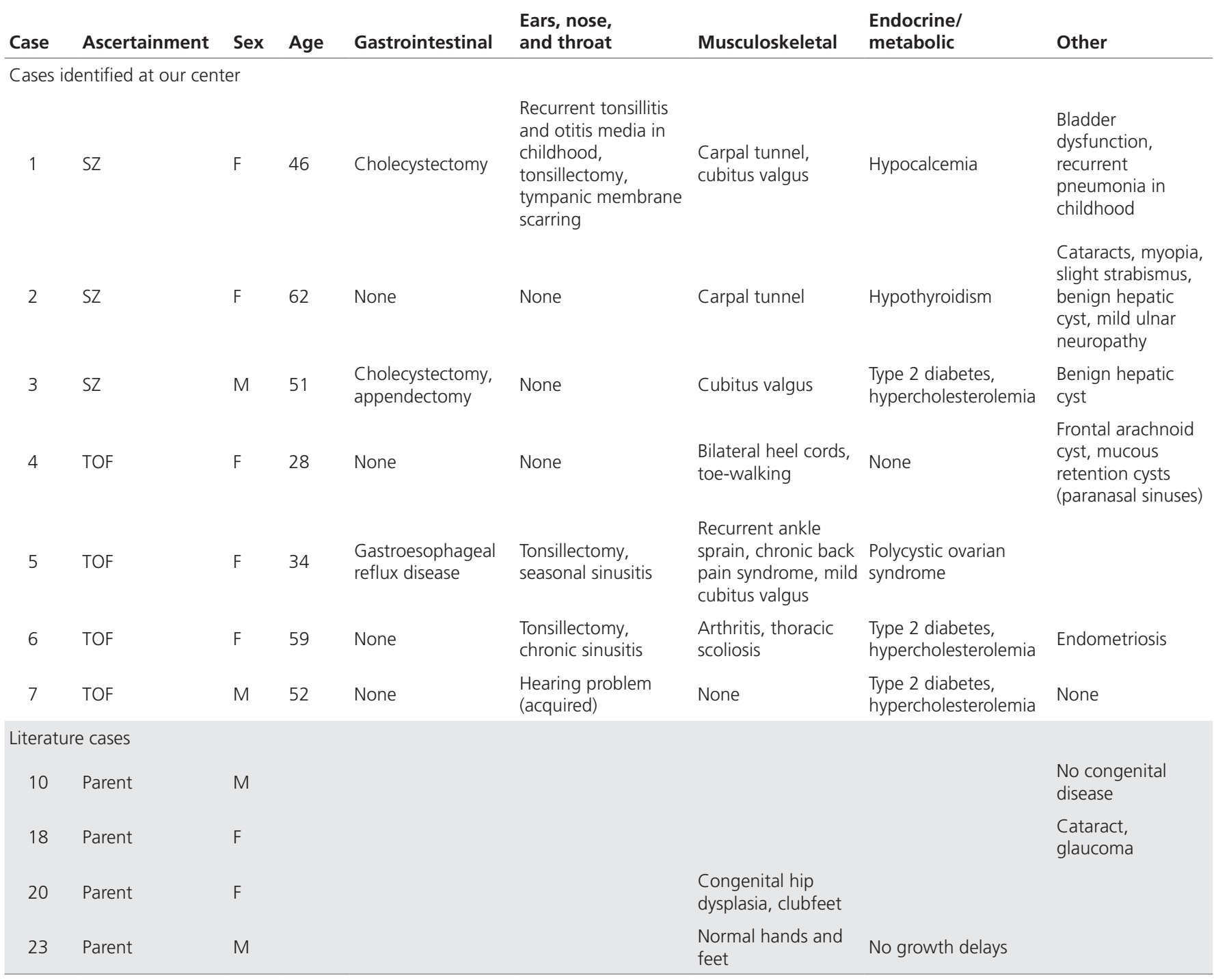

No further clinical information was available for literature cases 8, 9, 11-17, 19, 21-22, and 24-27 in Table 1.

Empty cells, unknown; F, female; M, male; SZ, schizophrenia; TOF, tetralogy of Fallot.

results had macrocephaly, similar to the proportion $(\sim 50 \%)$ reported in a previous study of 18 children with 1q21.1 duplications. ${ }^{1}$ Of 13 adults with any data on growth parameters, nine had one or more of three features (macrocephaly, tall stature, and/or obesity) suggestive of a possible overgrowth phenotype. Eight subjects had macrocephaly, four of whom had tall stature and/or obesity. Six of the 13 adults had no data on height or weight. Furthermore, we identified a tentative association with abnormalities of possible connective tissue origin, such as cysts, varicose veins, carpal tunnel syndrome, and congenital hip dysplasia. Overall, phenotypes of adults showed perhaps fewer differences from those of children than expected, as head circumference results were similar and $25 \%$ of adults with data had learning disabilities, as compared with $\sim 15 \%$ of the general population. More severe features such as intellectual disability or autism that would have been detectable in children and would be expected to persist into adulthood were rarer in the adults reported, consistent with our hypothesis of a milder phenotype in adults with 1q21.1 duplication as compared with children.

\section{Implications}

Anticipatory care of patients with 1q21.1 duplications should include monitoring for late-onset conditions that may be associated, including schizophrenia, mood disorders, and perhaps abnormalities of connective tissue origin. Given the association between 1q21.1 duplications and congenital cardiac disease (specifically TOF), newly diagnosed patients, including adults, may also warrant echocardiogram investigation. However, the extent of the variable expressivity and incomplete penetrance of 1q21.1 duplications remains largely unknown as yet. Family studies and further clinical data using standardized assessments will be essential to characterize these genetic parameters and to inform anticipatory care of affected individuals. 
GJA5 is the primary candidate gene reported for the congenital cardiac phenotype of 1q21.1 duplication syndrome. ${ }^{5,37}$ There are now four subjects with TOF reported to have small duplications overlapping GJA5: three $100-200 \mathrm{~kb}^{5}$ and one $10.3 \mathrm{~kb}^{37}$ in size. GJA5 encodes connexin 40, a cardiac gap junction protein expressed in the right ventricular outflow tract. ${ }^{10}$ Evidence from UCSC gene expression data suggests that genes involved in the neuropsychiatric phenotype of 1q21.1 duplications may include $B C L 9, G J A 8, P D Z K 1$, and PRKAB2. ${ }^{40}$ Previously proposed for the macrocephaly phenotype is a 1q21.1 region paralog of the HYDIN gene on chromosome 16q22.2, which is exclusively expressed in the brain and has been implicated in hydrocephalus. ${ }^{1}$ Other possibilities may need to be considered, however, if general overgrowth and connective tissue abnormalities are associated.

\section{Advantages and limitations}

This is the first study to focus on a review of adults with 1q21.1 duplications. An advantage to studying this age group is the potential to determine whether late-onset conditions, including major psychiatric disorders such as schizophrenia, and other disorders are associated. Limitations to our study were the relatively small sample size currently available for review and the paucity of detailed phenotypic data. Studies reporting "normal" phenotypes often provided no details about specific assessment tools. Access to large, well-characterized samples, likely involving international consortia, will be needed to further delineate the lifetime phenotype and the contributing genotype of individuals with 1q21.1 duplications. Moreover, a lack of genome-wide data meant that we could not comment on other rare structural variants that could contribute to and/ or modify expression in individuals with 1q21.1 duplications.

\section{Conclusions}

In summary, the 1q21.1 duplication is significantly enriched in populations of patients with major developmental conditions, including TOF and schizophrenia. A greater proportion of adults as compared with children were female, possibly due to differences in ascertainment. In addition to learning difficulties, the expected variability of the associated phenotype appears to include macrocephaly and perhaps other features of overgrowth, as well as abnormalities of connective tissue origin. More information on lifetime clinical features of individuals with 1q21.1 duplications is needed to inform genetic counseling and anticipatory care.

\section{SUPPLEMENTARY MATERIAL}

Supplementary material is linked to the online version of the paper at http://www.nature.com/gim

\section{ACKNOWLEDGMENTS}

This study was supported by Canadian Institutes of Health Research grants (MOP-89066, MOP-53216, and MOP-97800) and a University of Toronto Excellence Award in the Social Sciences and Humanities (to A.D.). A.S.B. holds the Canada Research Chair in Schizophrenia Genetics and Genomic Disorders. The authors thank the patients and their families for their participation, research assistants and staff at the Toronto Congenital Cardiac Centre for Adults, staff at The Centre for Applied Genomics, and fellows and students who assisted in the collection and analysis of data.

\section{DISCLOSURE}

The authors declare no conflict of interest.

\section{REFERENCES}

1. Brunetti-Pierri N, Berg JS, Scaglia F, et al. Recurrent reciprocal 1q21.1 deletions and duplications associated with microcephaly or macrocephaly and developmental and behavioral abnormalities. Nat Genet 2008;40:14661471.

2. Mefford HC, Sharp AJ, Baker C, et al. Recurrent rearrangements of chromosome 1q21.1 and variable pediatric phenotypes. N Engl J Med 2008;359:1685-1699.

3. Kaminsky EB, Kaul V, Paschall J, et al. An evidence-based approach to establish the functional and clinical significance of copy number variants in intellectual and developmental disabilities. Genet Med 2011;13:777-784.

4. Cooper DN, Bacolla A, Férec C, Vasquez KM, Kehrer-Sawatzki H, Chen JM. On the sequence-directed nature of human gene mutation: the role of genomic architecture and the local DNA sequence environment in mediating gene mutations underlying human inherited disease. Hum Mutat 2011;32:10751099.

5. Soemedi R, Topf A, Wilson IJ, et al. Phenotype-specific effect of chromosome 1q21.1 rearrangements and GJA5 duplications in 2436 congenital heart disease patients and 6760 controls. Hum Mol Genet 2012;21:1513-1520.

6. International Schizophrenia Consortium. Rare chromosomal deletions and duplications increase risk of schizophrenia. Nature 2008;455:237-241.

7. Harvard C, Strong E, Mercier E, et al. Understanding the impact of 1q21.1 copy number variant. Orphanet J Rare Dis 2011;6:54

8. Weber S, Landwehr C, Renkert M, et al. Mapping candidate regions and genes for congenital anomalies of the kidneys and urinary tract (CAKUT) by array-based comparative genomic hybridization. Nephrol Dial Transplant 2011;26:136-143.

9. Levinson DF, Duan J, Oh S, et al. Copy number variants in schizophrenia: confirmation of five previous findings and new evidence for 3 q29 microdeletions and VIPR2 duplications. Am J Psychiatry 2011;168:302-316.

10. Greenway SC, Pereira AC, Lin JC, et al. De novo copy number variants identify new genes and loci in isolated sporadic tetralogy of Fallot. Nat Genet 2009;41:931-935.

11. Pinto $D$, Pagnamenta AT, Klei $L$, et al. Functional impact of global rare copy number variation in autism spectrum disorders. Nature 2010;466:368-372.

12. Brunet $A$, Armengol $L$, Heine $D$, et al. BAC array $C G H$ in patients with Velocardiofacial syndrome-like features reveals genomic aberrations on chromosome region 1q21.1. BMC Med Genet 2009;10:144

13. Weiss LA. Autism genetics: emerging data from genome-wide copynumber and single nucleotide polymorphism scans. Expert Rev Mol Diagn 2009;9:795-803.

14. Bassett AS, Scherer SW, Brzustowicz LM. Copy number variations in schizophrenia: critical review and new perspectives on concepts of genetics and disease. Am J Psychiatry 2010;167:899-914.

15. Vissers LE, de Vries BB, Veltman JA. Genomic microarrays in mental retardation: from copy number variation to gene, from research to diagnosis. J Med Genet 2010;47:289-297

16. Breckpot J, Thienpont B, Arens $Y$, et al. Challenges of interpreting copy number variation in syndromic and non-syndromic congenital heart defects. Cytogenet Genome Res 2011;135:251-259.

17. Kirov G, Grozeva D, Norton N, et al.; International Schizophrenia Consortium; Wellcome Trust Case Control Consortium. Support for the involvement of large copy number variants in the pathogenesis of schizophrenia. Hum Mol Genet 2009;18:1497-1503.

18. Stefansson H, Rujescu D, Cichon S, et al.; GROUP. Large recurrent microdeletions associated with schizophrenia. Nature 2008;455:232-236.

19. Szatmari P, Paterson AD, Zwaigenbaum L, et al.; Autism Genome Project Consortium. Mapping autism risk loci using genetic linkage and chromosomal rearrangements. Nat Genet 2007;39:319-328.

20. Breckpot J, Thienpont B, Peeters H, et al. Array comparative genomic hybridization as a diagnostic tool for syndromic heart defects. J Pediatr 2010;156:810-7, 817.e1. 
21. Zogopoulos G, Ha KC, Naqib F, et al. Germ-line DNA copy number variation frequencies in a large North American population. Hum Genet 2007;122: 345-353.

22. Sanders SJ, Ercan-Sencicek AG, Hus V, et al. Multiple recurrent de novo CNVs, including duplications of the 7q11.23 Williams syndrome region, are strongly associated with autism. Neuron 2011;70:863-885

23. Redon $\mathrm{R}$, Ishikawa $\mathrm{S}$, Fitch $\mathrm{KR}$, et al. Global variation in copy number in the human genome. Nature 2006;444:444-454.

24. Shaikh TH, Gai X, Perin JC, et al. High-resolution mapping and analysis of copy number variations in the human genome: a data resource for clinical and research applications. Genome Res 2009;19:1682-1690.

25. Shaffer LG, Kashork CD, Saleki R, et al. Targeted genomic microarray analysis for identification of chromosome abnormalities in 1500 consecutive clinical cases. J Pediatr 2006;149:98-102.

26. Weiss LA, Shen Y, Korn JM, et al.; Autism Consortium. Association between microdeletion and microduplication at 16p11.2 and autism. N Engl J Med 2008:358:667-675

27. de Vries BB, Pfundt $R$, Leisink $M$, et al. Diagnostic genome profiling in mental retardation. Am J Hum Genet 2005;77:606-616.

28. Bucan M, Abrahams BS, Wang K, et al. Genome-wide analyses of exonic copy number variants in a family-based study point to novel autism susceptibility genes. PLoS Genet 2009;5:e1000536.

29. Grozeva D, Kirov G, Ivanov D, et al.; Wellcome Trust Case Control Consortium. Rare copy number variants: a point of rarity in genetic risk for bipolar disorder and schizophrenia. Arch Gen Psychiatry 2010;67:318-327.

30. Rucker JJ, Breen G, Pinto D, et al. Genome-wide association analysis of copy number variation in recurrent depressive disorder. Mol Psychiatry 2011; e-pub ahead of print 1 November 2011.
31. Hehir-Kwa JY, Rodríguez-Santiago B, Vissers LE, et al. De novo copy number variants associated with intellectual disability have a paternal origin and age bias. J Med Genet 2011:48:776-778.

32. Cooper GM, Coe BP, Girirajan S, et al. A copy number variation morbidity map of developmental delay. Nat Genet 2011;43:838-846.

33. Malhotra D, McCarthy S, Michaelson JJ, et al. High frequencies of de novo CNVs in bipolar disorder and schizophrenia. Neuron 2011;72:951-963.

34. Glessner JT, Reilly MP, Kim CE, et al. Strong synaptic transmission impact by copy number variations in schizophrenia. Proc Natl Acad Sci USA 2010;107:1058410589.

35. Sahoo T, Theisen A, Rosenfeld JA, et al. Copy number variants of schizophrenia susceptibility loci are associated with a spectrum of speech and developmental delays and behavior problems. Genet Med 2011;13:868880.

36. Bushby KM, Cole T, Matthews JN, Goodship JA. Centiles for adult head circumference. Arch Dis Child 1992;67:1286-1287.

37. Silversides CK, Lionel AC, Costain G, et al. Rare copy number variations in adults with tetralogy of fallot implicate novel risk gene pathways. PLoS Genet 2012:8:e1002843

38. Fung WL, Chow EW, Webb GD, Gatzoulis MA, Bassett AS. Extracardiac features predicting 22q11.2 deletion syndrome in adult congenital heart disease. Int $\rfloor$ Cardiol 2008;131:51-58.

39. Lionel AC, Crosbie J, Barbosa N, et al. Rare copy number variation discovery and cross-disorder comparisons identify risk genes for ADHD. Sci Trans/ Med 2011:3:95ra75.

40. Bassett AS, Lionel AC, Costain $G$, et al. The burden of rare copy number variants in community-based schizophrenia suggests a potential role for clinical microarrays. In submission. 